\title{
Serial galectin-3 and future cardiovascular disease in the general population - Data of PREVEND
}

\author{
A. Rogier van der Velde, MD*, Wouter C. Meijers, ${ }^{\star}{ }^{\star}$, Jennifer E. Ho, MD, $\mathrm{PhD}^{\dagger}$, Frank $\mathbf{P}$. \\ Brouwers, MD, PhD ${ }^{\star}$, Michiel Rienstra, MD, PhD ${ }^{\star}$, Stephan J.L. Bakker, MD, PhD ${ }^{\ddagger}$, Anneke \\ C. Muller Kobold, MD, PhD§, Dirk J. van Veldhuisen, MD, PhD*, Wiek van Gilst, MD, PhD*, \\ Pim van der Harst, MD, PhD $^{\star}$, and Rudolf A. de Boer, MD, $\mathrm{PhD}^{\star}$
}

\begin{abstract}
*University of Groningen, University Medical Center Groningen, Groningen, Department of Cardiology, the Netherlands ${ }^{\dagger}$ Cardiovascular Medicine Section, Department of Medicine, Boston University School of Medicine, Boston, USA ¥University of Groningen, University Medical Center Groningen, Department of Nephrology, Groningen, the Netherlands §University of Groningen, University Medical Center Groningen, Department of Laboratory Medicine, Groningen, the Netherlands
\end{abstract}

\begin{abstract}
Background-Lifetime risk for cardiovascular (CV) disease is high but predicting incident events on an individual level remains difficult. Single measurements of galectin-3, a marker of tissue fibrosis, predict mortality and new-onset heart failure (HF). Persistently elevated levels may indicate a clinically silent disease process.

Objectives-Our aim was to establish the value of serial galectin-3 measurements to predict CV outcomes in the general population.

Methods-Plasma galectin-3 was measured in the PREVEND study at baseline and after $\sim 4$ years. Changes in serial galectin-3 were expressed as categorical changes or absolute change from baseline and were related to subsequent outcome.

Results-Serial galectin-3 was measured in 5,958 subjects (mean age 49 \pm 12 years; $49 \%$ female). The median duration of follow-up was 8.3 years. Persistently elevated galectin-3 (defined as highest quartile at baseline and highest quartile during visit $2, \mathrm{n}=757$ subjects) was associated with a higher risk for new-onset HF, CV mortality, all-cause mortality, new-onset atrial fibrillation and CV events, compared to subjects with non-persistently elevated galectin-3. After multivariable adjustments for baseline characteristics, serial galectin-3 remained an independent predictor of
\end{abstract}

Correspondence to: Rudolf A. de Boer, MD PhD FESC, University of Groningen, Department of Cardiology, Medical Center Groningen, PO Box 30.001, 9700 RB, Groningen, the Netherlands, Phone: +31 503612355 / Fax: +31 503615525 / r.a.de.boer@umcg.nl.

The Corresponding Author has the right to grant on behalf of all authors and does grant on behalf of all authors, an exclusive licence (or non exclusive for government employees) on a worldwide basis to the BMJ Publishing Group Ltd and its Licensees to permit this article (if accepted) to be published in HEART editions and any other BMJPGL products to exploit all subsidiary rights

DISCLOSURES

Dr. van Veldhuisen reported receiving board membership fees and/or travel expenses from Amgen, Johnson \& Johnson, Novartis, Sorbent, Vifor, Cardiorentis, and BioControl. The other authors do not report disclosures. 
new-onset HF (Hazard ratio (HR) 1.85[1.10-3.13];P=0.02) but not for other outcomes. Serial measurements provided more accurate prognostic value to predict new-onset $\mathrm{HF}$, compared to a single baseline measurement (Harrell's C: 0.72[0.68-0.75] versus 0.68[0.65-0.72]; $\mathrm{P}=0.002$, respectively) with significant net reclassification (NRI), however, the incremental value was low.

Conclusions-Persistently elevated galectin-3 predicts new-onset HF after adjustment for covariates, and serial measurements provide more accurate prognostic information compared to single determination of galectin-3. This may help to identify individuals who are at risk for incident $\mathrm{HF}$ and might provide a measure to monitor interventions.

\section{Keywords}

biomarker; galectin-3; risk prediction; heart failure; healthy ageing

\section{INTRODUCTION}

Risk prediction in cardiovascular (CV) disease can be helpful to provide lifestyle advice and to guide clinical decision-making. However, it remains a challenge to predict the risk of the individual subjects for incident events [1]. In clinical practice, biomarkers may be useful to improve the accuracy of prognostic evaluation and may be used to detect increased risk for progression to heart failure (HF) or any other $\mathrm{CV}$ event during early stages of disease [2]. Although single baseline biomarker levels provide additive value to existing clinical models, it has been proposed that serial biomarker measurements may be more powerful than single measurements [3].

In recent years, galectin-3 has emerged as a prognostic marker for CV disease [4]. A metaanalysis comprising 8,419 patients reported the independent prognostic value of galectin-3 in patients with HF [5]. Galectin-3 is a beta-galactoside binding lectin and a mediator of tissue fibrosis and inflammation; processes that are known to play an important role in the development and progression of CV disease [4]. Galectin-3 levels are low in healthy hearts but are increased in hearts which are at risk to develop HF [6]. In addition to its role as a prognostic biomarker, galectin-3 may also serve as a target for pharmacological interventions in $\mathrm{CV}$ disease $[7,8]$.

Whether repeated biomarker assessment can add to single biomarker assessments in order to predict long-term outcomes has not been well established for galectin-3. From a biological standpoint, an assessment at a given time point reflects a certain status at a given time point, and therefore serial measurements could be useful, since it contains more information.

Single galectin-3 measurement predicts outcome in patients with acute and chronic HF [911], and is associated with mortality and development of HF in patients with atherosclerosis $[12,13]$ and in the general population, we and other groups have shown that single galectin-3 measurements can be helpful to identify patients who are at risk for development of HF, CV events and all-cause mortality [14-16], Further, serial measurements of galectin-3 provide additive prognostic value in patients with prevalent HF [17]. But in the general population, all of the studies reported prognostic value of a single galectin-3 measurement. Our aim was to report the prognostic value of serial galectin-3 measurements in the general population in prediction of future $\mathrm{CV}$ events and to compare its value with single measurements. 


\section{METHODS}

\section{Study patient cohort: PREVEND study}

The Prevention of REnal and Vascular ENd-stage Disease (PREVEND) is a large cohort $(n=8,592)$ derived from the general population. Details of the study protocol have been described in detail elsewhere [15,18]. Blood was drawn and anticoagulated with EDTA and stored at $-80^{\circ} \mathrm{C}$. The first follow-up visit took place after a median of 4.2 [interquartile range: 4.0-4.5] years. Non-caucasian subjects were excluded $(n=379)$ because we cannot assume normal ranges of galectin-3 established in Caucasians are the same in all ethnicities. Subjects with new-onset HF before first follow-up visit $(n=41)$, HF present at baseline $(\mathrm{n}=11)$, missing samples at baseline $(\mathrm{n}=245)$ or follow-up $(\mathrm{n}=1958)$ and subjects who died between the two measurements $(n=417)$ were excluded from the analysis. The PREVEND study was approved by the institutional medical Ethics Committee and was conducted in accordance with the declaration of Helsinki. All subjects have provided written informed consent.

\section{Measurement of galectin-3}

Galectin-3 levels were measured at baseline and follow-up using an enzyme-linked immunosorbent assay (ELISA; BG Medicine, Inc., Waltham, MA, USA). Intra- and interassay coefficients of variation of this assay are $3.2 \%$ and $5.6 \%$, respectively, and the lower limit of detection is $1.13 \mathrm{ng} / \mathrm{mL}$ [19]. The samples of baseline were measured in duplicate (average values were used in our analysis); samples at follow-up in singular.

\section{Definitions and outcome variables}

Definitions of baseline and outcome variables have been described previously [20,21]. The follow-up time of the present study ranged from the second follow-up visit to December 31, 2010 or event if this occurred before this date. Subjects were censored on the dates of either moving away to an unknown location or last study visit, whichever occurred first. Changes in galectin-3 concentration were assessed by 3 different methods: 1) To assess the prognostic value of high galectin-3 levels over time, participants were categorized in two groups: patients with persistently elevated galectin-3 levels and patients with non-persistently elevated galectin-3 levels. We defined galectin-3 levels above the third quartile as elevated, so the category of persistently elevated galectin-3 level identifies patients with galectin-3 levels above the third quartile at baseline and above the third quartile at their follow-up visit (Q4-Q4). Whereas the category of non-persistently elevated galectin-3 levels identifies all other patients who are not in Q4-Q4. 2) To study the effect of changes in galectin-3, subjects were divided into 4 groups, based on changes in galectin-3 levels from baseline to follow-up across a threshold level. This level was calculated as the concentration of galectin-3 that yielded the highest youden-index (highest product of sensitivity and specificity) for experiencing new-onset $\mathrm{HF}$, which were $12.2 \mathrm{ng} / \mathrm{mL}$ at baseline and $14.6 \mathrm{ng} / \mathrm{mL}$ at followup. 3) Furthermore, in an additional sensitivity analysis, we studied the prognostic value of absolute change in galectin-3 over time, which was also categorized in 4 equal quartiles. 


\section{Statistical analyses}

Normally distributed variables are presented as means \pm standard deviation (SD). Nonnormally distributed variables are expressed as medians [interquartile range (IQR)] and were log-transformed before regression analyses. Clinical and biochemical characteristics were compared across two groups using two-sample t-test for continuous, normally distributed variables, Wilcoxon rank-sum test for continuous, non-normally distributed variables and chi-square test for categorical variables. Baseline characteristics were also compared across four groups using ANOVA for continuous, normally distributed variables, Kruskal-Wallis test for continuous, non-normally distributed variables and chi-square test for categorical variables. To correct for years of sample storage, a linear correction coefficient for the follow-up galectin-3 measurements was applied, for each age category. The PREVEND trial was set up to overselect participants with increased UAE (>10 mg/L). A design-based statistical weighting was used to adjust for this overselection allowing conclusions to be made for the general population.

Time to event between different groups, based on galectin-3 levels over time, was compared using cumulative incidence curves and Wald chi-square tests. To account for death as competing risk for other outcomes, subjects' follow-up time was censored at the time of death whenever death occurred before the onset of outcome. The association between serial galectin-3 with several endpoints was studied employing competing risk regression based on a proportional hazards model using hazard ratios as measures of association, with $95 \%$ confidence intervals based on robust standard error estimates. The model was adjusted for all baseline characteristics except for the covariates that showed collinearity (cholesterol, LDL, HDL, creatinine, cystatin-C).

The prognostic discrimination of a single determination of galectin-3 compared to serial measurements of galectin-3 was assessed by the Harrell's C index and 95\% confidence intervals (CIs) were calculated using the method of Newson to compare the predictive power of survival models, as described previously [22]. We furthermore estimated the integrated discrimination improvement (IDI) and the category-free net reclassification improvement (NRI) for the addition of baseline or serial Gal-3 in fully adjusted models $P<0.05$ (twosided) was used as level of statistical significance. All analyses were performed using Stata/MP (version 14.0).

\section{RESULTS}

\section{Study population and baseline characteristics}

Baseline characteristics of 5,958 subjects are presented in Table 1 for subjects with persistently elevated (defined as galectin-3 levels in the $4^{\text {th }}$ quartile at both time points (Q4Q4) and non-persistently elevated galectin-3 (not Q4-Q4). Characteristics of participants that were excluded in the present study are presented in supplemental table 1. Median [IQR] follow-up time of the present study was 8.3 [7.8-8.9] years from the first follow-up visit. The median galectin-3 level at baseline was 10.8 [9.0-13.0] and increased to 11.5 [9.0-14.4] after $\sim 4$ years of follow-up and was higher in female (baseline: 11.0 [9.1-13.3]; follow-up: 11.7 [9.1-14.8]) compared to male subjects (10.6 [8.9-12.6]; 11.3 [8.9-14.0]; $\mathrm{P}<0.0001$; 
Figure 1). Subjects with persistently high galectin-3 levels (Q4-Q4) had an elevated CV risk: they were older, were more often female, were less likely to smoke, had more frequently diabetes mellitus type 2, a higher prevalence of MI, hypertension, hypercholesterolemia and stroke, a higher blood pressure, a lower eGFR and had higher levels of cholesterol, triglycerides, glucose, creatinine, UAE, hs-CRP, NT-proBNP and galectin-3 (Table 1). The etiology of heart failure was similar to subjects with non-persistently elevated galectin-3 levels (Supplemental Table 2).

We also studied changes of galectin-3 over time using our defined optimized cut points at baseline $(12.2 \mathrm{ng} / \mathrm{mL})$ and at follow-up $(14.6 \mathrm{ng} / \mathrm{mL})$; baseline characteristics of these categories are presented in supplemental table 3 . To further explore the effects of galectin-3 dynamics, we also show the absolute change in galectin-3, and were also categorized by quartiles of absolute change in galectin-3 (Supplemental Table 4). Patients with the highest increase of galectin-3 over a $\sim$-year time period were older, had more often hypertension and hypercholesterolemia, lower eGFR, higher NT-proBNP and lower galectin-3 at baseline.

\section{Galectin-3 levels over time and event rate}

All subjects were followed for a mean duration of $\sim 8$ years after their first follow-up measurement. A total of 352 individuals died, with 86 due to CV causes. Furthermore, 188 subjects experienced new-onset HF (of which 73 subjects with heart failure with preserved ejection fraction (HFpEF) and 115 subjects with heart failure with reduced ejection fraction (HFrEF), 537 a CV event and 225 participants experienced new-onset AF. Baseline and follow-up galectin-3 levels were classified into quartiles. The association between serial galectin-3 levels and events is depicted in $4 \times 4$ heat plots of serial galectin-3 levels with its corresponding event rate (Figure 2). Subjects with persistently high galectin-3 levels $\left(4^{\text {th }}\right.$ quartile at baseline and $4^{\text {th }}$ quartile at follow-up) over time have the highest event rate for CV mortality, new-onset HF, all-cause mortality, new-onset AF and CV events (Figure 2; Supplemental Figure 1-2).

In order to visualize and calculate the differences between the persistently elevated subjects in comparison to the pooled non-persistently elevated subjects, cumulative incidence curves were plotted which showed that individuals with persistently elevated galectin-3 levels, compared to non-persistently elevated, demonstrate a higher CV mortality (Wald chi-square test: $\mathrm{P}<0.0001)$, higher cumulative incidence of $\mathrm{HF}(\mathrm{P}<0.0001)$ (Figure 3$)$ and also a higher all-cause mortality $(\mathrm{P}<0.0001)$, higher cumulative incidence of new-onset $\mathrm{AF}(\mathrm{P}=0.005)$ and $\mathrm{CV}$ events $(\mathrm{P}<0.0001)$ in patients with persistently elevated galectin-3 levels (Supplemental Figure 3).

We conducted secondary analysis using an optimized cut point creating four groups (lowlow, low-high, high-low and high-high). Analysis of categorical change of galectin-3 over time showed the highest event rate in the "high-high group" for all endpoints (Figure 4; Supplemental figure 4). Furthermore, the association between absolute change of galectin-3 over time and outcome was studied to determine whether the level of change in galectin-3 was important. Cumulative incidence curves, by quartile of absolute change in galectin-3, showed that the absolute change in galectin-3 was associated with increased CV mortality (Wald chi-square test: $\mathrm{P}=0.02)$, all-cause mortality $(\mathrm{P}=0.01)$ and $\mathrm{CV}$ events $(\mathrm{P}=0.04)$, but not 
with new-onset HF ( $\mathrm{P}=0.24$; Supplemental Figure 5). Subjects with the largest increase in galectin-3 had low galectin-3 levels at baseline (10.4 ng/mL; Supplemental Figure 6).

\section{Independent predictive value of serial galectin-3 levels}

The independent predictive value of serial galectin-3 levels over a $\sim 4$-year time period, was assessed with competing-risks regression models, and included adjustment for all baseline characteristics (Table 2). In an univariable analysis, persistently elevated galectin-3 levels were associated with CV mortality, new-onset HF, all-cause mortality, new-onset atrial fibrillation and CV event (Table 2, Supplemental Table 5), compared to subjects with nonpersistently elevated galectin-3 levels. In age- and sex- adjusted analyses, persistently elevated galectin-3 levels were still associated with an increased risk for new-onset HF and CV mortality (Table 2, Supplemental Table 5). After multivariable adjustment for all baseline characteristics, serial elevated galectin-3 levels remained an independent predictor for new-onset HF, while the predictive value for CV mortality was lost. The independent association of persistently elevated galectin-3 with new-onset HF was primarily attributed to new-onset HFrEF while HFpEF was not significantly predicted, although there was no statistical significant interaction (Supplemental Table 6; 6 subjects had an EF between 40\% and $50 \%$ and were excluded from this sub analysis).

A secondary analysis showing the multivariable analysis of categorical change over time confirmed that subjects in the "high-high" group impose an increased risk for new-onset HF, while the risk for all-cause mortality was also significantly increased after multivariable adjustment in this analysis (Table 3), while in a tertiary multivariable competing-risks regression analysis of absolute change of galectin-3, which was adjusted for baseline galectin-3 levels, the largest increases in galectin-3 were only associated with all-cause mortality (Supplemental Table 7).

\section{Predictive value of serial measurements compared to single measurement}

To assess the predictive value of serial measurements over single measurements for prediction of CV endpoints, Harrell's C index was calculated (Table 4). This index was assessed for a single measurement at baseline, a single measurement at follow-up and as a serial measurement at baseline and follow-up. In a direct comparison with a single measurement, serial measurement of galectin-3 had the greatest prognostic accuracy for $\mathrm{CV}$ death, new-onset HF, new-onset $\mathrm{AF}$ and $\mathrm{CV}$ event, but not for all-cause mortality.

\section{DISCUSSION}

We demonstrate in a large cohort of the general population, that persistently elevated galectin-3 levels predict new-onset HF, more accurately than a single measurement. In a comparison of persistently elevated versus non-persistently elevated galectin-3 levels, all end points were univariably associated with serial galectin-3 levels, and this association was still present for new-onset HF after full adjustment, although lost for other end points. In a secondary analysis, using low-high categories for a defined cut point; high galectin-3 levels over time were independently associated with new-onset HF and all-cause mortality. Finally, as a third strategy, we studied the association of absolute changes in galectin-3 over time 
with outcome. Using this strategy of analysis, we observed that, after correction for baseline galectin-3 level, larger absolute increases in galectin-3 levels over time were not independently associated with a higher risk for future CV disease. Subjects with the highest absolute increase in galectin-3 had low galectin-3 levels (10.4 ng/mL) at baseline, which might explain the overall limited risk. Apparently, in the general population, persistent elevated levels of galectin-3 portend stronger risk than increasing galectin-3.

In our study, persistently elevated galectin-3 levels over time were compared to nonpersistently elevated galectin-3 levels. We selected patients with galectin-3 in the highest quartiles, representing "elevated" values, which clearly impose the highest risk. This approach was chosen, instead of using a distinct cut-off; each cohort will have its own distinct galectin-3 levels, while a $4^{\text {th }}$ quartile will be applicable to every cohort. As a secondary analysis, we studied change over time using a defined cut point, categorizing patients from "high" to "low" galectin-3 levels to assess the prognostic value of increasing and decreasing galectin-3 levels across a defined cut point. Furthermore, to assess the importance of the magnitude of increase in galectin-3, we studied absolute changes of galectin-3.

Collectively, serial galectin-3 levels show borderline predictive value for (all cause, CV) mortality in the general population: some of our analyses suggested a predictive value for all-cause and CV mortality, but these observations were more consistent for all-cause mortality, compared to CV mortality. The multivariable analysis reaffirmed that sex and especially age are important confounders of galectin-3, as was shown previously [23]. Altogether these results indicate that serial galectin-3 levels are particularly useful to predict new-onset $\mathrm{HF}$ and possibly mortality, but is of no use to predict new-onset AF and CV events in the general population, as was already suggested by single measurements of galectin-3 [24].

Prediction of CV disease in the general population remains a challenge, although recent previous research has identified several predictors of new-onset HF[20,25]. Baseline biomarkers including age, NT-proBNP, the presence of diabetes mellitus and a history of valvular disease have been shown to predict overall new-onset HF[25]. Whether repeated measurements of prognostic predictors are useful in prediction of outcome in CV disease remains topic of discussion. Biomarkers might reflect underlying changes in pathophysiology and therefore serial measurements might give additional information about the current status of disease. For instance, a substudy of the Val-HeFT trial (Valsartan Heart Failure Trial) showed that serial determinations of NT-proBNP might be a superior strategy for risk stratification of chronic and stable HF patients [26]. However, each biomarker has its distinct variability over time and can be more or less sensitive to changes in the present disease status.

Galectin-3 is a biomarker that has shown to be rather stable over time and is not affected by volume loading status [27]. Interestingly, data from four independent cohorts of prevalent HF have strongly suggested that serial measurements show prognostic value in diseased patients $[17,28,29]$. Also in ostensibly healthy subjects, single measurements of galectin-3 have shown to be predictive for outcome [14-16]. In this present study, we now extend these 
findings by showing that serial galectin-3 measurements in the general population are associated with adverse outcome. Previous studies have already suggested a role for serial galectin-3 measurements to guide therapy, because of its low biological variation [30]. Further, it is well known that fibrosis in cardiac remodeling is a slow phenomenon [8]. Our results can be seen in line with this notion. A short-term increase in galectin-3 therefore, measured by a single measurement, may not be sufficient to induce profound differences. However, long-lasting elevated levels of galectin-3, obtained by serial measurements, may detect subclinical CV disease.

Collectively, our results show that persistently elevated galectin-3 is an independent predictor of new-onset HF in the general population. Taken into account these results, serial sampling of galectin-3 can help the clinician to identify apparently healthy subjects who are at risk for development of HF at an early stage. Whether these subjects are amenable to specific pharmacotherapy should be tested. Finally, subjects with lower galectin-3 levels over time can be regarded as low-risk for development of HF.

\section{Study limitations}

By design, the PREVEND trial overselected subjects with micro-albuminuria. Therefore we applied a statistical weighting method to correct for this so that we were able to extend our conclusions to the general population. Because we study serial measurements, there is a potential risk for survivorship bias, because patients that died between the visits are excluded from the analysis. In our analyses we tried to minimize survivorship bias, by conducting a competing-risks regression.

\section{Acknowledgments}

\section{SOURCES OF FUNDING}

Dr. de Boer was supported by the Innovational Research Incentives Scheme program of the Netherlands Organization for Scientific Research [Vidi grant 917.13.350]. Dr. M. Rienstra is supported by a grant from the Netherlands Organization for Scientific Research (Veni grant 016.136.055). Dr. Ho is supported by the National Institutes of Health (K23-HL116780) and a Boston University School of Medicine, Department of Medicine Career Investment Award.

\section{ABBREVIATIONS}

$\begin{array}{ll}\text { AF } & \text { Atrial fibrillation } \\ \text { ANOVA } & \text { Analysis of variance } \\ \text { BMI } & \text { Body mass index } \\ \text { BP } & \text { Blood pressure } \\ \text { CV } & \text { Cardiovascular } \\ \text { CABG } & \text { Coronary artery bypass grafting } \\ \text { EDTA } & \text { Ethylenediaminetetraacetic acid } \\ \text { eGFR } & \text { Estimated glomerular filtration rate }\end{array}$




\begin{tabular}{ll} 
ELISA & Enzym-linked immunosorbent assay \\
ESC & European society of cardiology \\
HDL & High-density lipoprotein \\
HF & Heart failure \\
HR & Hazard ratio \\
Hs-CRP & High-sensitivity C-reactive protein \\
IQR & Interquartile range \\
LDL & Low-density lipoprotein \\
MI & Myocardial infarction \\
MRA & Mineralocorticoid receptor antagonist \\
NT-proBNP & N-terminal pro-B-type natriuretic peptide \\
PCI & Percutaneous coronary intervention \\
SD & Standard deviation \\
SMDRD & Simplified modification of diet in renal disease \\
UAE & Urinary albumin excretion \\
Val-HeFT & Valsartan heart failure trial \\
\hline
\end{tabular}

\section{References}

1. Ouwerkerk W, Voors AA, Zwinderman AH. Factors influencing the predictive power of models for predicting mortality and/or heart failure hospitalization in patients with heart failure. JACC Heart Fail. 2014; 2:429-36. DOI: 10.1016/j.jchf.2014.04.006 [PubMed: 25194294]

2. de Couto G, Ouzounian M, Liu PP. Early detection of myocardial dysfunction and heart failure. Nat Rev Cardiol. 2010; 7:334-44. DOI: 10.1038/nrcardio.2010.51 [PubMed: 20458341]

3. Januzzi JL, Troughton R. Are serial BNP measurements useful in heart failure management? Serial natriuretic peptide measurements are useful in heart failure management. Circulation. 2013; 127:500-7. discussion 508. DOI: 10.1161/CIRCULATIONAHA.112.120485 [PubMed: 23357662]

4. de Boer RA, Voors AA, Muntendam P, et al. Galectin-3: a novel mediator of heart failure development and progression. Eur J Heart Fail. 2009; 11:811-7. DOI: 10.1093/eurjhf/hfp097 [PubMed: 19648160]

5. Chen A, Hou W, Zhang Y, et al. Prognostic value of serum galectin-3 in patients with heart failure: A meta-analysis. Int J Cardiol. 2015; 182:168-70. DOI: 10.1016/j.ijcard.2014.12.137 [PubMed: 25577755]

6. Sharma UC, Pokharel S, van Brakel TJ, et al. Galectin-3 marks activated macrophages in failureprone hypertrophied hearts and contributes to cardiac dysfunction. Circulation. 2004; 110:3121-8. DOI: 10.1161/01.CIR.0000147181.65298.4D [PubMed: 15520318]

7. Yu L, Ruifrok WPT, Meissner M, et al. Genetic and pharmacological inhibition of galectin-3 prevents cardiac remodeling by interfering with myocardial fibrogenesis. Circ Heart Fail. 2013; 6:107-17. DOI: 10.1161/CIRCHEARTFAILURE.112.971168 [PubMed: 23230309] 
8. de Boer RA, van der Velde AR, Mueller C, et al. Galectin-3: a modifiable risk factor in heart failure. Cardiovasc Drugs Ther. 2014; 28:237-46. DOI: 10.1007/s10557-014-6520-2 [PubMed: 24789662]

9. van Kimmenade RR, Januzzi JL, Ellinor PT, et al. Utility of amino-terminal pro-brain natriuretic peptide, galectin-3, and apelin for the evaluation of patients with acute heart failure. J Am Coll Cardiol. 2006; 48:1217-24. DOI: 10.1016/j.jacc.2006.03.061 [PubMed: 16979009]

10. de Boer RA, Lok DJA, Jaarsma T, et al. Predictive value of plasma galectin-3 levels in heart failure with reduced and preserved ejection fraction. Ann Med. 2011; 43:60-8. DOI: 10.3109/07853890.2010.538080 [PubMed: 21189092]

11. Lok DJA, Van Der Meer P, de la Porte PWB-A, et al. Prognostic value of galectin-3, a novel marker of fibrosis, in patients with chronic heart failure: data from the DEAL-HF study. Clin Res Cardiol. 2010; 99:323-8. DOI: 10.1007/s00392-010-0125-y [PubMed: 20130888]

12. Drechsler C, Delgado G, Wanner C, et al. Galectin-3, Renal Function, and Clinical Outcomes: Results from the LURIC and 4D Studies. J Am Soc Nephrol. Published Online First: 7 January 2015.

13. Grandin EW, Jarolim P, Murphy SA, et al. Galectin-3 and the development of heart failure after acute coronary syndrome: pilot experience from PROVE IT-TIMI 22. Clin Chem. 2012; 58:26773. DOI: 10.1373/clinchem.2011.174359 [PubMed: 22110019]

14. Ho JE, Liu C, Lyass A, et al. Galectin-3, a marker of cardiac fibrosis, predicts incident heart failure in the community. J Am Coll Cardiol. 2012; 60:1249-56. DOI: 10.1016/j.jacc.2012.04.053 [PubMed: 22939561]

15. de Boer RA, van Veldhuisen DJ, Gansevoort RT, et al. The fibrosis marker galectin-3 and outcome in the general population. J Intern Med. 2012; 272:55-64. DOI: 10.1111/j. 1365-2796.2011.02476.x [PubMed: 22026577]

16. Jagodzinski A, Havulinna AS, Appelbaum S, et al. Predictive value of galectin-3 for incident cardiovascular disease and heart failure in the population-based FINRISK 1997 cohort. Int J Cardiol. 2015; 192:33-9. DOI: 10.1016/j.ijcard.2015.05.040 [PubMed: 25985013]

17. van der Velde AR, Gullestad L, Ueland T, et al. Prognostic value of changes in galectin-3 levels over time in patients with heart failure: data from CORONA and COACH. Circ Heart Fail. 2013; 6:219-26. DOI: 10.1161/CIRCHEARTFAILURE.112.000129 [PubMed: 23395934]

18. Diercks GF, Janssen WM, van Boven AJ, et al. Rationale, design, and baseline characteristics of a trial of prevention of cardiovascular and renal disease with fosinopril and pravastatin in nonhypertensive, nonhypercholesterolemic subjects with microalbuminuria (the Prevention of REnal and Vascular E. Am J Cardiol. 2000; 86:635-8. [accessed 8 Jan 2015] http:// www.ncbi.nlm.nih.gov/pubmed/10980214. [PubMed: 10980214]

19. Meijers WC, van der Velde AR, de Boer RA. The ARCHITECT galectin-3 assay: comparison with other automated and manual assays for the measurement of circulating galectin-3 levels in heart failure. Expert Rev Mol Diagn. 2014; 14:257-66. http://www.ncbi.nlm.nih.gov/pubmed/24606321. [PubMed: 24606321]

20. Brouwers FP, de Boer RA, van der Harst P, et al. Incidence and epidemiology of new onset heart failure with preserved vs. reduced ejection fraction in a community-based cohort: 11-year followup of PREVEND. Eur Heart J. 2013; 34:1424-31. DOI: 10.1093/eurheartj/eht066 [PubMed: 23470495]

21. Vermond RA, Geelhoed B, Verweij N, et al. Incidence of Atrial Fibrillation and Relationship With Cardiovascular Events, Heart Failure, and Mortality: A Community-Based Study From the Netherlands. J Am Coll Cardiol. 2015; 66:1000-7. DOI: 10.1016/j.jacc.2015.06.1314 [PubMed: 26314526]

22. Shlipak MG, Weekley CC, Li Y, et al. Comparison of cardiovascular prognosis by 3 serum cystatin $\mathrm{C}$ methods in the Heart and Soul Study. Clin Chem. 2011; 57:737-45. DOI: 10.1373/clinchem. 2010.158915 [PubMed: 21310869]

23. Tang WHW, Shrestha K, Shao Z, et al. Usefulness of plasma galectin-3 levels in systolic heart failure to predict renal insufficiency and survival. Am J Cardiol. 2011; 108:385-90. DOI: 10.1016/ j.amjcard.2011.03.056 [PubMed: 21600537]

24. Ho JE, Yin X, Levy D, et al. Galectin 3 and incident atrial fibrillation in the community. Am Heart J. 2014; 167:729-34. e1. DOI: 10.1016/j.ahj.2014.02.009 [PubMed: 24766984] 
25. Ho JE, Lyass A, Lee DS, et al. Predictors of new-onset heart failure: differences in preserved versus reduced ejection fraction. Circ Heart Fail. 2013; 6:279-86. DOI: 10.1161/ CIRCHEARTFAILURE.112.972828 [PubMed: 23271790]

26. Masson S, Latini R, Anand IS, et al. Prognostic value of changes in N-terminal pro-brain natriuretic peptide in Val-HeFT (Valsartan Heart Failure Trial). J Am Coll Cardiol. 2008; 52:9971003. DOI: 10.1016/j.jacc.2008.04.069 [PubMed: 18786480]

27. Milting H, Ellinghaus P, Seewald M, et al. Plasma biomarkers of myocardial fibrosis and remodeling in terminal heart failure patients supported by mechanical circulatory support devices. J Heart Lung Transplant. 2008; 27:589-96. DOI: 10.1016/j.healun.2008.02.018 [PubMed: 18503956]

28. Anand IS, Rector TS, Kuskowski M, et al. Baseline and serial measurements of galectin-3 in patients with heart failure: relationship to prognosis and effect of treatment with valsartan in the Val-HeFT. Eur J Heart Fail. 2013; 15:511-8. DOI: 10.1093/eurjhf/hfs205 [PubMed: 23291728]

29. Motiwala SR, Szymonifka J, Belcher A, et al. Serial measurement of galectin-3 in patients with chronic heart failure: results from the ProBNP Outpatient Tailored Chronic Heart Failure Therapy (PROTECT) study. Eur J Heart Fail. 2013; 15:1157-63. DOI: 10.1093/eurjhf/hft075 [PubMed: 23666680]

30. Wu AHB, Wians F, Jaffe A. Biological variation of galectin-3 and soluble ST2 for chronic heart failure: implication on interpretation of test results. Am Heart J. 2013; 165:995-9. DOI: 10.1016/ j.ahj.2013.02.029 [PubMed: 23708172] 


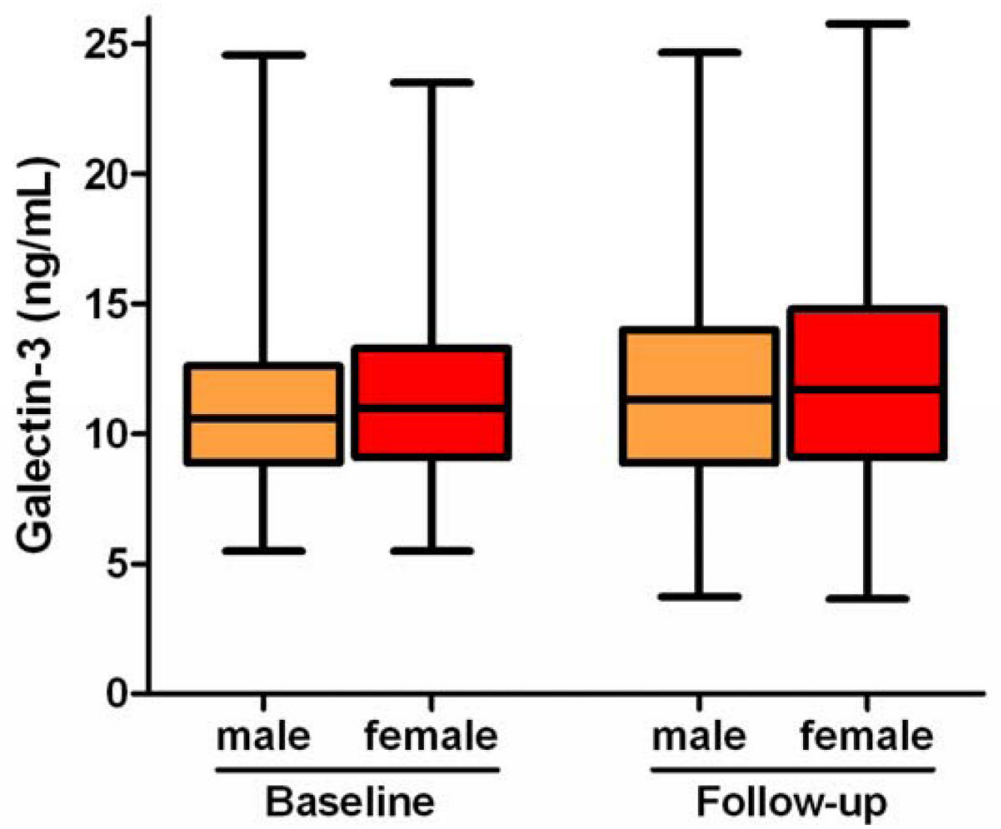

Figure 1.

Box-and-whisker plot showing the plasma galectin-3 levels $(\mathrm{ng} / \mathrm{mL})$ at baseline and at follow-up in male and female subjects. The boxplot represents median and interquartile range and whiskers represent the $1^{\text {st }}$ and $99^{\text {th }}$ percentile. 


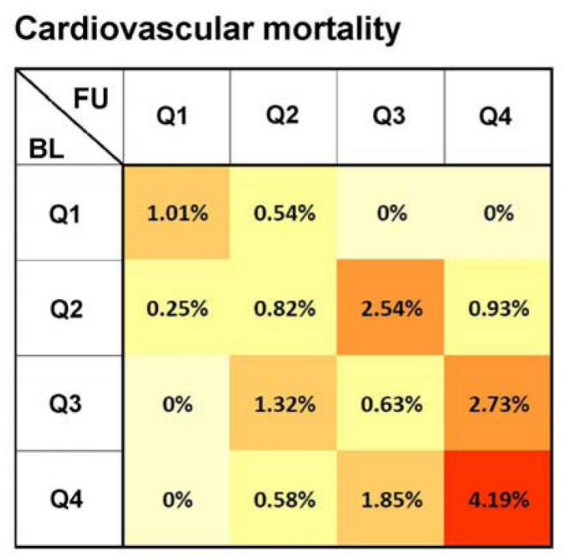

New-onset heart failure

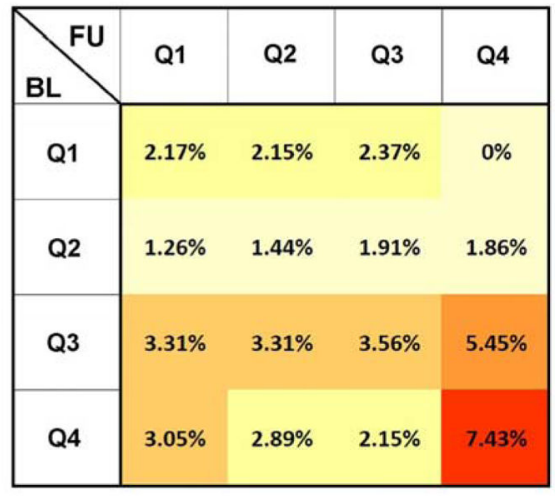

Figure 2.

$4 \times 4$ Heat plots showing the relative event rate for cardiovascular mortality and new-onset heart failure, categorized by quartile of galectin-3 at baseline (BL) and at follow-up (FU). Intervals of galectin-3 quartiles at baseline are: Q1 (3.9-9.0); Q2 (9.1-10.8); Q3 (10.913.0); Q4 (13.1-77.7). Intervals of galectin-3 quartiles at follow-up are: Q1 (3.0-8.9); Q2 (9.0-11.5); Q3 (11.6-14.4); Q4 (14.5-74.1). Subjects with galectin-3 levels in the $4^{\text {th }}$ quartile at both time points have the highest event rate. 

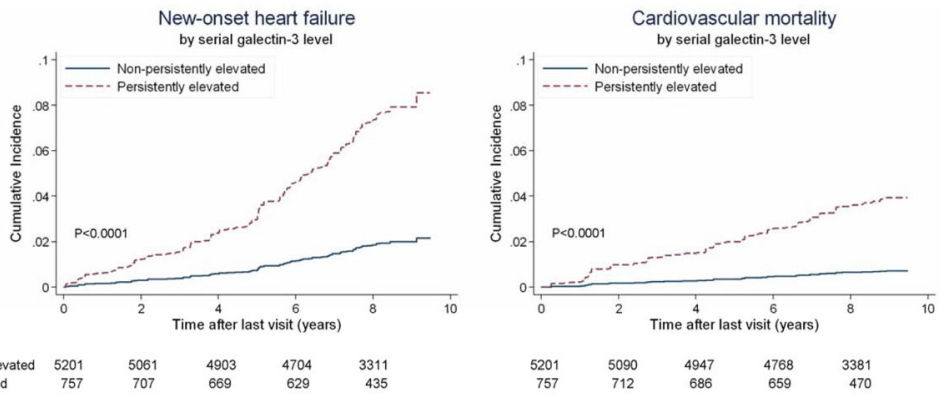

Figure 3.

Cumulative incidence curves showing CV mortality and new-onset HF by persistently and non-persistently elevated galectin-3 levels. Subjects showed increased CV mortality and cases of new-onset HF with persistently elevated galectin-3. 

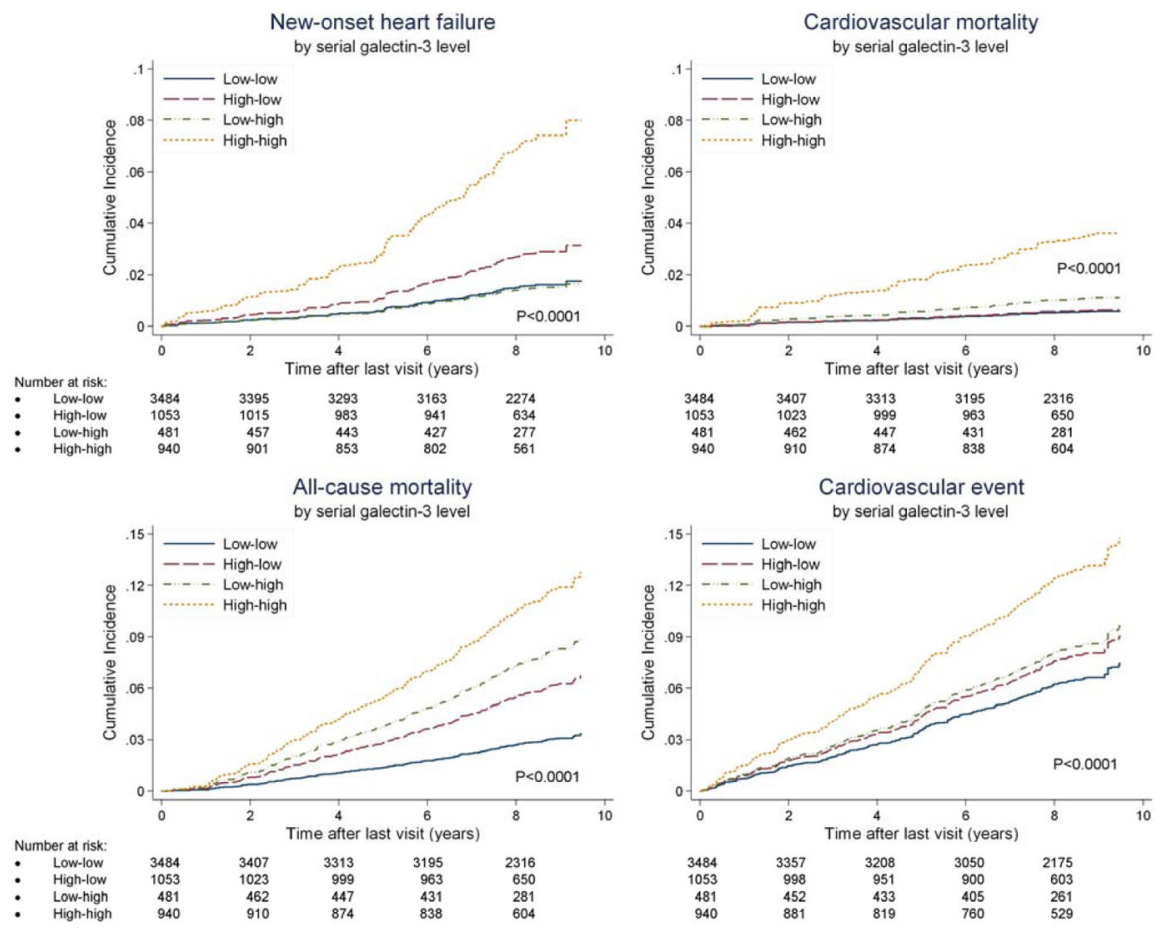

Figure 4.

Cumulative incidence curves showing CV mortality, new-onset HF, all-cause mortality and $\mathrm{CV}$ event by categorical change of galectin- 3 . 


\section{Table 1}

Baseline characteristics by serial galectin-3 level

\begin{tabular}{|c|c|c|c|c|}
\hline & Total & Non-persistently elevated Galectin-3 & Persistently elevated Galectin-3 & $\boldsymbol{P}$ \\
\hline $\mathrm{N}$ & 5958 & 5201 & 757 & \\
\hline Age (years) & $49.1(12.0)$ & $47.9(11.6)$ & $56.9(12.0)$ & $<0.001$ \\
\hline Gender (male), $\mathrm{N}(\%)$ & $2940(49.3 \%)$ & $2625(50.5 \%)$ & $315(41.6 \%)$ & $<0.001$ \\
\hline Smoking (last 5 years), $\mathrm{N}(\%)$ & $2558(43.2 \%)$ & $2281(44.1 \%)$ & $277(36.7 \%)$ & $<0.001$ \\
\hline Diabetes mellitus, $\mathrm{N}(\%)$ & $67(1.1 \%)$ & $48(0.9 \%)$ & $19(2.5 \%)$ & $<0.001$ \\
\hline Myocardial Infarction, N(\%) & $138(2.3 \%)$ & $102(2.0 \%)$ & $36(4.8 \%)$ & $<0.001$ \\
\hline Hypertension, $\mathrm{N}(\%)$ & $1516(25.4 \%)$ & $1222(23.5 \%)$ & $294(38.8 \%)$ & $<0.001$ \\
\hline Hypercholesterolemia, $\mathrm{N}(\%)$ & $1548(26.3 \%)$ & $1259(24.5 \%)$ & $289(38.7 \%)$ & $<0.001$ \\
\hline Stroke, $\mathrm{N}(\%)$ & $42(0.7 \%)$ & $29(0.6 \%)$ & $13(1.7 \%)$ & $<0.001$ \\
\hline Waist-hip-ratio & $0.9(0.1)$ & $0.9(0.1)$ & $0.9(0.1)$ & $<0.001$ \\
\hline BMI $\left(\mathrm{kg} \mathrm{m}^{-2}\right)$ & $26.0(4.1)$ & $25.8(4.0)$ & $27.2(4.6)$ & $<0.001$ \\
\hline Systolic BP (mm Hg) & $128.2(19.3)$ & $127.1(18.6)$ & $135.4(22.3)$ & $<0.001$ \\
\hline Diastolic BP (mm Hg) & $73.7(9.6)$ & $73.5(9.5)$ & $75.5(9.9)$ & $<0.001$ \\
\hline Cholesterol $\left(\mathrm{mmol} \mathrm{L}^{-1}\right)$ & $5.6[4.9-6.3]$ & $5.5[4.8-6.3]$ & $5.9[5.2-6.7]$ & $<0.001$ \\
\hline $\operatorname{LDL}\left(\mathrm{mmol} \mathrm{L}^{-1}\right)$ & $3.6[2.9-4.3]$ & $3.6[2.9-4.3]$ & $3.9[3.2-4.6]$ & $<0.001$ \\
\hline $\operatorname{HDL}\left(\mathrm{mmol} \mathrm{L}^{-1}\right)$ & $1.3[1.0-1.6]$ & $1.3[1.0-1.6]$ & $1.2[1.0-1.5]$ & 0.020 \\
\hline Triglycerides $\left(\mathrm{mmol} \mathrm{L}^{-1}\right)$ & $1.1[0.8-1.7]$ & $1.1[0.8-1.6]$ & $1.4[1.0-1.9]$ & $<0.001$ \\
\hline Glucose $\left(\mathrm{mmol} \mathrm{L}^{-1}\right)$ & $4.7[4.3-5.1]$ & $4.7[4.3-5.1]$ & $4.8[4.4-5.3]$ & $<0.001$ \\
\hline Creatinine $\left(\mu \mathrm{mol} \mathrm{L}^{-1}\right)$ & $82.0[74.0-91.0]$ & $82.0[73.0-91.0]$ & $84.0[75.0-96.0]$ & $<0.001$ \\
\hline Cystatin-C $\left(\mathrm{mg} \mathrm{L}^{-1}\right)$ & $0.8[0.7-0.9]$ & $0.8[0.7-0.8]$ & $0.8[0.7-1.0]$ & $<0.001$ \\
\hline eGFR $\left(\mathrm{mL} \min ^{-1}\right)$ & 79.8 [71.4-88.9] & 80.7 [72.6-89.7] & $72.1[63.9-82.0]$ & $<0.001$ \\
\hline UAE (mg per $24 \mathrm{~h}$ ) & $9.1[6.3-15.9]$ & $9.1[6.3-15.4]$ & $9.4[6.2-19.8]$ & 0.093 \\
\hline hs-CRP $\left(\mathrm{mg} \mathrm{L}^{-1}\right)$ & $1.2[0.5-2.8]$ & $1.1[0.5-2.7]$ & $1.8[0.9-3.9]$ & $<0.001$ \\
\hline NT-proBNP $\left(p g \mathrm{~mL}^{-1}\right)$ & $36.7[16.3-70.3]$ & $34.8[15.4-65.7]$ & $52.7[24.4-105.1]$ & $<0.001$ \\
\hline Galectin-3 (ng/mL) & $10.8[9.0-13.0]$ & $10.3[8.8-12.0]$ & $15.4[14.1-17.5]$ & $<0.001$ \\
\hline
\end{tabular}

BMI: body mass index; BP: blood pressure; cholesterol: total cholesterol; LDL: low-density lipoprotein cholesterol; HDL: high-density lipoprotein cholesterol; creatinine: serum creatinine; eGFR: estimated glomerular filtration rate, according to the Modification of Diet in Renal Disease formula; UAE: urinary albumin excretion rate; hs-CRP: high-sensitivity C-reactive protein; NT-proBNP: N-terminal pro-B-type natriuretic peptide. 

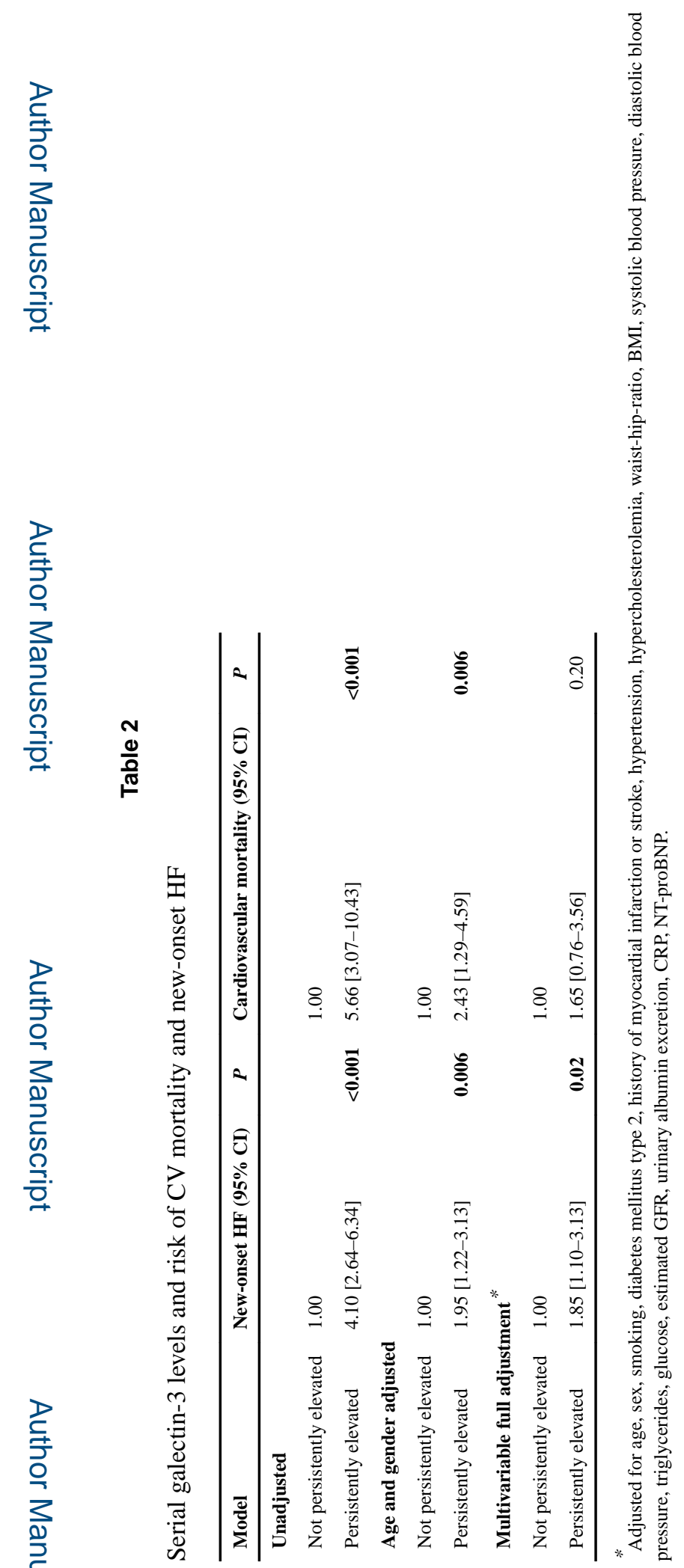

Heart. Author manuscript; available in PMC 2017 July 15. 
van der Velde et al.

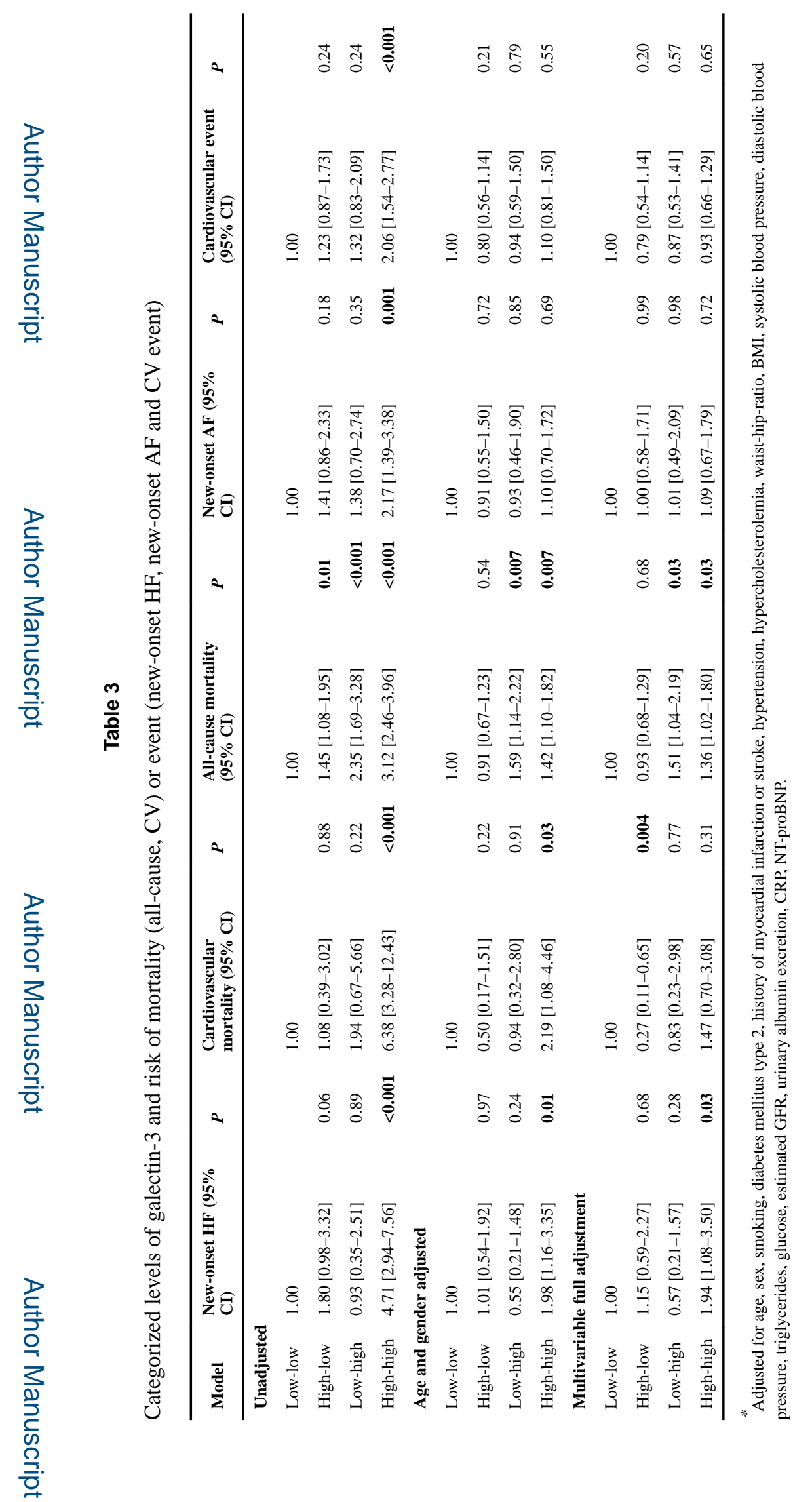

Heart. Author manuscript; available in PMC 2017 July 15. 


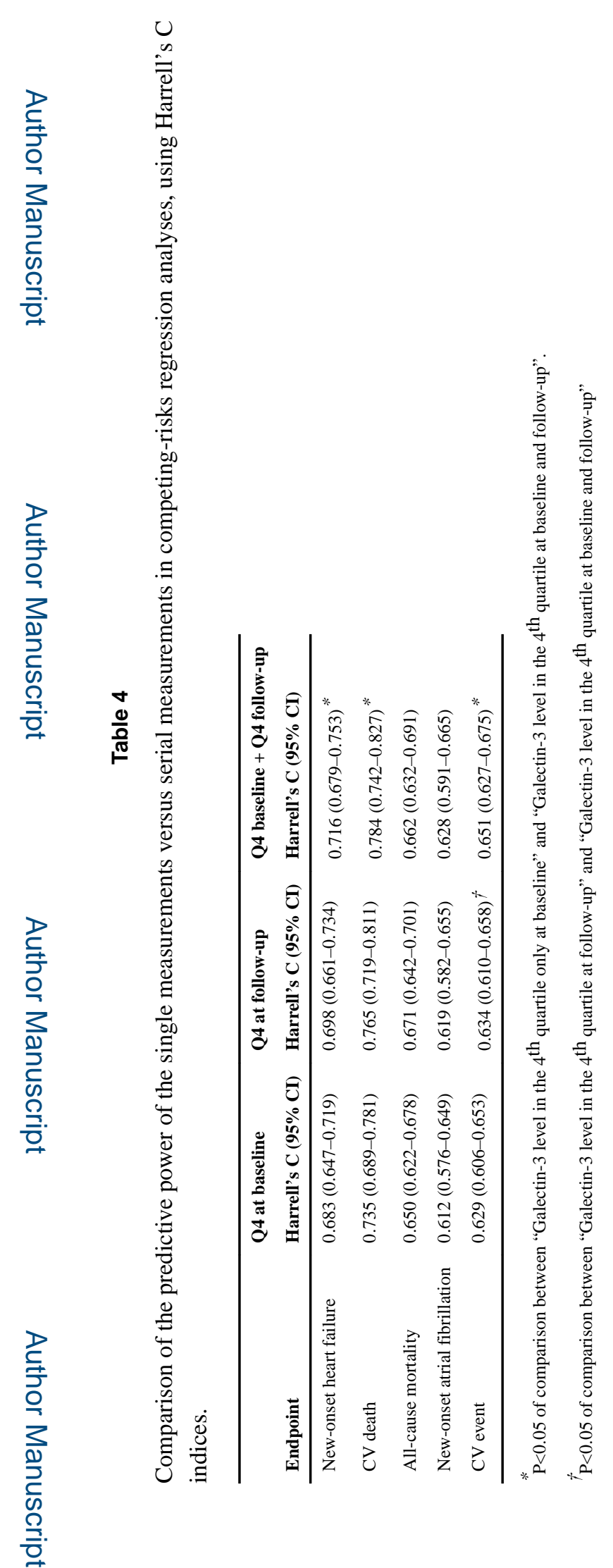

Heart. Author manuscript; available in PMC 2017 July 15. 